\title{
Impact of COVID-19 on Stomatology Professionals
}

\section{Edurner FPA ${ }^{1}$, Carmen HSCL ${ }^{2 *}$ and Beatriz LSSP ${ }^{3}$}

${ }^{1}$ Department of Stomatology, Universidad Señor de Sipán, Spain

${ }^{2}$ Biologist MINSA-Executive Directorate of Environmental Health, Spain

${ }^{3}$ Full-time Professor, School of Stomatology, Universidad Señor de Sipán, Spain

*Corresponding author: Henckell Sime Clara Luisa del Carmen, Biologist MINSA-Executive Directorate of Environmental Health, Spain, Email: claryh@hotmail.com

\section{Research Article}

Volume 5 Issue 4

Received Date: December 03, 2020

Published Date: December 10, 2020

DOI: $10.23880 /$ oajds- 16000283

\section{Abstract}

The current state of pandemic caused by the SARS-CoV-2 virus, commonly called the "new coronavirus" that causes the CoVID-19 disease, has generated a great impact in the dental field, since the characteristics that it possesses condition a permanent state of alert by health professionals due to its high transmissibility of positive patients, even asymptomatic, or in the recovery phase, which makes it highly contagious; Thanks to a precarious health system, high rates of mortality and contagion have been revealed; That is why the present research aims to determine the repercussions of the CoVID-19 disease in stomatologists during dental practice; A search was carried out in the databases of the USS campus and academic google and 21 scientific articles were chosen under the inclusion criteria that evidence studies on CoVID-19, in Spanish and English; excluding those who did not contribute to the investigation, nor did they maintain the relationship with CoVID-19 and dentistry, through the technique of documentary analysis; All the information was collected in collection sheets whose criteria were summary and results; Within the last ones, it was evidenced that dentistry practice has been mainly affected by the present virus, because its transmission route is through the conjunctival, nasal and oral mucosa, the purpose of the dentist being to preserve oral health at the mucosal level present in the oral cavity; Likewise, another point that generates high contagion potential is dental instruments, which cause droplets of saliva to be produced, or even blood; It concludes with the call to raise awareness of the degree of virus contagion exposure to which the stomatologist is exposed.

Keywords: COVID-19; Stomatologist; Oral medicine

Abbreviations: ICTRP: International Clinical Trials Registry Platform; SARS: Severe Acute Respiratory Syndrome; PPE: Personal Protective Equipment.

\section{Introduction}

The recent outbreak of the "new coronavirus" has generated a state of health emergency worldwide. The first sign of this virus was on December 1,2019, when an unknown beta coronavirus was discovered in a group of people with pneumonia who frequented the wholesale seafood market in Wuhan, China. Coronaviruses are enveloped RNA viruses that are widely distributed among humans, other mammals, and birds, causing respiratory, enteric, liver, and neurological diseases [1]. The current pandemic has forced the various world and local entities in charge of preserving human health to be present, establishing stricter protection measures at the individual level and at the population level, each person carrying their respective primary protection barrier, manifesting in this point the mandatory use of the mask in the entire Peruvian population and from the population perspective, maintaining as stated by entities and protocols a minimum of 1.5 to 2 meters of distance between person and person; in the labor context its impact was imminent and the field of the stomatologist was no exception, we refer to the service of dental health practice that is provided to the 
patient, because the virus is transmitted through our mucous membranes, it leads to high possibilities of contracting it and until now it has not been successful in determining with precision an effective treatment against CoVID-19, therefore in the field of dental professionals, it is important to be diligent, since the devices to be used generate aerosols, increasing exponentially the risk of infection and therefore, having an impact on the way of providing the stomatological service. The obvious changes in outpatient care involve increasing the time in preparing the care for the same, as well as an additional cost in personal protective equipment for the patient and an additional cost in supplies such as thermometer, alcohol and bleach by the professional providing care. The general objective of this review was to determine the impact of CoVID-19 on dental professionals, so two specific objectives were taken into account: Describe the potential risk of contagion in the dental field and identify the protection measures that the stomatologist in his professional practice.

\section{Methodology}

The research was carried out through a qualitative, exploratory design, since the subject investigated examines our current health emergency situation due to covid-19, which is a little-studied problem, use was made of databases located on the virtual campus of the USS; such as EBSCO, ProQuest, ScienceDirect, Scielo, ResearchGate, recommended for their scientific and editorial quality, as well as the Journal of Oral Research, Academic google, in which 25 scientific sources on covid-19 were reviewed, of which they were chosen 17 having as inclusion criteria: articles that evidence studies of COVID-19, in the languages: Spanish and English, and exclusion: those that do not contribute to the research, nor touch the relationship between COVID-19 and dental practice.

To collect the information, the document analysis technique was used and all the information was stored by means of the data collection sheet instrument, in which the information from each consulted bibliographic source was collected and organized; according to criteria such as summary and results; recording each data electronically in Excel.

\section{Results}

\section{Coronavirus Overview}

The Orthocoronaviridae, known as coronaviruses, are one of the subfamilies belonging to the Coronaviridae, with phylogenetic similarities to single-stranded RNA viruses, with a helical nucleocapsid and crown-shaped tips on the surface of the virus; for this reason they are called coronavirus [2]; the coronaviruses are grouped into 4 genera, the Alphacoronavirus, betacoronavirus, Gammacoronavirus and Deltacoronavirus; In the last seventeen years, studies of the betacoronavirus genus are of great importance for the scientific community and for the health sector, since two of them have been the cause of severe acute respiratory syndrome (SARS-CoV) and that of respiratory syndrome of the Middle East (MERS-CoV) [3]. As mentioned previously, there are four genera of coronavirus (Table 1), within each genus there are subgenera and species, of which the Alphacoronavirus and Betacoronavirus have bats as host and Gammacoronavirus covers recognized avian types [2] (Figure 1).

\begin{tabular}{|lll|}
\hline Género & Subgéneros & Especies \\
\hline $\begin{array}{l}\text { Alfacoronavirus, } \\
\text { antes Coronavirus } \\
\text { grupo } 1 \text { (CoV-1) }\end{array}$ & 17 especies \\
\hline $\begin{array}{l}\text { Betacoronavirus, } \\
\text { antes como subgéneros } \\
\text { Coronavirus grupo } \\
2 \text { (CoV-2) }\end{array}$ & 11 especies \\
\hline Deltacoronavirus & 4 subgéneros & 7 especies \\
\hline Gammacoronavirus & 2 subgéneros & 2 especies \\
\hline
\end{tabular}

Source: Taken from the College of Physiotherapists of the community of Madrid.

Figure 1: Coronavirus Genres.

\begin{tabular}{|c|c|c|}
\hline Authors & \multicolumn{2}{|c|}{ Recommendations } \\
\hline $\begin{array}{c}\text { Collegiate } \\
\text { Organization of } \\
\text { Dentists of Spain }\end{array}$ & $\begin{array}{c}\text { Dental } \\
\text { consultation }\end{array}$ & $\begin{array}{c}\text { He recommends carrying out the virtual consultation, taking an anamnesis whose } \\
\text { questions rule out possible positive COVID-19 cases. }\end{array}$ \\
\hline $\begin{array}{c}\text { Dental College of } \\
\text { Peru }\end{array}$ & Patient care & $\begin{array}{c}\text { Strict instructions must be followed before, during and after each care; As prior to } \\
\text { taking the temperature, disinfecting hands, footwear, such as clothing, wearing their } \\
\text { respective personal protective equipment, after the care the dentist must disinfect and } \\
\text { sterilize their respective instruments used, before attending the next patient (5). }\end{array}$ \\
\hline
\end{tabular}

Table 1: Recommendations during dental practice against COVID-19. 


\section{Open Access Journal of Dental Sciences}

\section{COVID-19 Overview}

The recent coronavirus causes severe acute respiratory syndrome, manifesting for the first time atypical pneumonia in the month of December 2019, in Wuhan, Hubei province, China [4] which has been named COVID-19. China openly shared information about the gene sequence of this virus on January 12, 2020, through the gisaid.org platform [5]. The first case outside of China was detected in Thailand, on January 13,2020 , because a person had travelled to Wuhan; in subsequent days, more cases were detected in more Asian countries such as Japan, South Korea; and on January 20 the first positive case in the US was confirmed, of a person who travelled from Wuhan to Washington. After the rapid transmission of Covid-19, the WHO declared a State of Public Health Emergency of International Importance (ESPII) (Public health emergency of international concern) and the same organization declared a state of pandemic on March 11 [6].

Due to the existing relationships between MERS-CoV and SARS-CoV, it is considered that the route of transmission of SARS-CoV-2 is through direct contact with the respiratory secretions of an infected animal or person, particularly through droplets from the cough, sneeze or mucous membranes of other people (nose, mouth or eyes) of less than $5 \mathrm{um}$, with an appearance of low probability of transmission by air at distances greater than 1 to 2 meters; the incubation period is variable, it is estimated to be approximately 4 to 7 days, with an average of 5 days, it is mentioned that the virus can infect in the incubation stage [7]. Recent research suggests that asymptomatic patients and those in the incubation period are also carriers of the virus, making its control too challenging due to the diffuse recognition of such patients, in the same way another powerful source of virus transmission is patients in a state of recovery [8].

A possibility of viability of aerosols and fomites is manifested, with a median life of SARS-CoV-2 of 6.8 hours in plastic, 5.6 hours in stainless steel, 3.5 hours in cardboard, 1.1 hours in aerosol, 0.8 hours in copper emphasizes its stability similar to that of SARS-CoV-1 [9]. The epidemiology and clinical results carried out in China on a sample of 41 patients who were positive for Covid-19 showed that, " $66 \%$ (27 patients) had direct contact with a large seafood and animal market. The average age was 49 years, with a male prevalence. Important signs and symptoms of 2019-nCoV were considered: fever (98\%), dry cough (76\%), dyspnea (55\%), myalgia or fatigue (44\%) and lymphopenia (63\%)" [10]. It is presumed that the origin of the virus transmission was due to the consumption in Wuhan of a bat soup, however, the presence of coronavirus has been evidenced in wild pangolins, presenting a $91 \%$ coincidence of the virus that causes COVID-19 [2]. In the study by Lam TT, et al. it is considered a zoonosis, based on the genetic sequencing of the virus, in bats and pangolin, considering it as the most probable origin of the virus [11]. According to Chan, et al. del Rio and Malani state that, "based on the findings of genetic and epidemiological research, it appears that the COVID-19 outbreak began with a single animal-to-human transmission, followed by a sustained spread from human to human.

A larger study conducted in Wuhan, China of 1,099 laboratory-confirmed positive patients from 552 hospitals, found the mean age to be 47 years; $41.9 \%$ were women; $5.0 \%$ were admitted to the ICU; $2.3 \%$ were on mechanical ventilation; 1 person died; $9 \%$ had previous contact with wildlife; $72.3 \%$ had contact with residents of Wuhan; with fever during admission of 43.8\%; during hospitalization $88.7 \%$ and $67.8 \%$ cough [12]. Symptoms range from mild to severe, appearing 2 to 14 days after exposure [13]. With regard to the incidence of covid-19, since December 2019, when the first positive case was confirmed, to date there are more than half a million people infected [13].

In Peru, the warning from the Medical College was towards the national health system, which has been overwhelmed by the number of patients, so hospitals are another source of virus transmission [14]. Until today, no cure or specific treatment for this virus has been found, but it is known that 382 clinical trials have been registered, 379 are registered in China, all information is in the International Clinical Trials Registry Platform (ICTRP) of the WHO, the most studied drugs that have shown some effect have been the non-specific (interferons) and specific antivirals (umifenovir, an HIV protease inhibitor, for combination with ritonavir and lopinavir); Antimalarial drugs are also being studied, such as chloroquine (shows some efficacy against HIV-AIDS), blocking SARS-CoV-2 infection in vitro, and hydroxychloroquine; corticosteroids can worsen covid-19 symptoms; inhaled gases (oxygen, hydrogen or their combination) give relief from hypoxia, molecular hydrogen is believed to be anti-inflammatory [15].

\section{Recommendations for Stomatologists against COVID-19}

At the level of dental practice, it is recommended to carry out a specific anamnesis of the patients, through five questions, it will be possible to identify aspects that determine a possible risk of contagion, the questions are, do you have a fever or have you had it in the last 14 days? Have you had a respiratory problem (including cough) in the last 14 days? Have you travelled to risk countries in the last 14 days? Have you been in contact with someone with confirmed coronavirus? Have you been in close contact with people who had acute respiratory symptoms in the last 14 days?; to be able to proceed with decision making [16]. 


\section{Open Access Journal of Dental Sciences}

In the same way, it is not considered that dental emergencies can occur, and due to the present pandemic, they are not going to stop attending to them, in view of this, rubber dams and high-volume saliva ejectors can contribute to reduce the aerosol or splashes in dental procedures, of course the use of biosafety elements by the dental professional and the patient [8]. It must also be taken into account that SARS-CoV-2 like other coronaviruses, has sensitivity to ultraviolet rays and heat, therefore, they can be inactivated with the application of lipid solvents that contain ether $(75 \%)$, ethanol, disinfectants (chlorine, peroxyacetic acid and chloroform), except chlorhexidine [15].

\section{Recommendations}

Collegiate Organization of Dentists of Spain Dental consultation. He recommends carrying out the virtual consultation, taking an anamnesis whose questions rule out possible positive COVID-19 cases. Dental College of Peru Patient care Strict instructions must be followed before, during and after each care; As prior to taking the temperature, disinfecting hands, footwear, such as clothing, wearing their respective personal protective equipment, after the care the dentist must disinfect and sterilize their respective instruments used, before attending the next patient [5].

Likewise, different organizations have called for a voluntary suspension of dental practice activities, reserving only those related to emergencies, but this leads us to reflect on the definition of "urgency". This pandemic forces us to redefine the meaning of what an "emergency in the stomatological field" means. Not only those defined in the formal documents of the institutions related to dentistry but also those "emergencies or needs felt by the population that require immediate treatment, such as, for example, the displacement of the arch in an orthodontic treatment that injures the cheeks; complications from ongoing endodontic treatments; perimplantitis, the dislodgement of crowns in the anterior dental sector; fracture of removable prostheses that prevent their use, people with disabilities and a long etcetera [17,18]. On the other hand, the Dental College of Peru has published a biosafety protocol for the dental surgeon during and after the pandemic; comments that upon confirmation of an emergency due to COVID-19, the person must communicate to 113 or the APP: "Peru in your hands."

Said protocol indicates that the use of a mask is mandatory for appointments, avoid crowding in the waiting room, the patient must attend alone or, given that they are a minor, a companion is not allowed on their own; comply with the social distance of at least 2 meters; not seeing patients without prior appointment; Before entering the dental center, the patient must wear a mask, alcohol must be applied to the footwear, put on boots, disinfect the hands with alcohol, wash hands, put on gloves; As mentioned above, the questioning is important, regardless of whether it is a suspected positive case, call 113 or the APP; clearly the whole environment has to be disinfected, materials sterilized, remove magazines, toys for children, water dispensers from the room; in small spaces maximum 2 people; in the waiting room avoid using cell phones; cleaning of the bathrooms with $0.1 \%$ faucet hypochlorite is recommended; brushing, prosthetics or removable appliances is prohibited in the bathroom of the dental center; The furniture, dental chair among others can be covered with plastic elements, these must be changed after each care; It should be understood that every patient is a potential carrier of covid-19, therefore before the procedure the patient should rinse his mouth for a minute with $0.5 \%$ $1 \%$ hydrogen peroxide, being careful to avoid burns, or with $0.2 \%$ povidone (after care with patients allergic to iodine), it can also be $0.05 \%-0.1 \%$ cetylpyridinium; the patient is totally forbidden to spit, therefore high power suction is used; If a procedure that originates aerosols must be carried out, an intermediate personal protective equipment (PPE) must be established, these are a hat, glasses, boots and apron for the patient; if halogen light lamps are used, the protection must be covered and changed when the patient changes; With regard to rotary instruments, these, when changing patients, must be disinfected and sterilized [5].

\section{Discussion}

The origin of the new coronavirus has several theories. For Professor Edward Holmes, from the University of Sydney, Australia [17], states, "that viruses that seem highly adapted to humans are present in wildlife. Bats are involved, pangolins perhaps, but it is possible that other species are also part of this chain. "However, in the study carried out by Lam TT, Shum MH, Zhu HC, Tong YG, Ni XB, Liao YS, et al; it is considered a zoonosis, based on the genetic sequencing of the virus, in bats and pangolin, considering it as the most probable origin of the virus [11]. According to Chan, et al. del Rio and Malani state that, Based on the findings of genetic and epidemiological research, it appears that the COVID-19 outbreak began with a single animal-to-human transmission, followed by a sustained human-to-human spread. For the purposes of this study, the new coronavirus is a natural part of wildlife and was transmitted by one of the animals that are natural reservoirs to man, from there it spread from person to person.

With regard to symptoms, "the epidemiology and clinical results carried out on a sample of 41 patients with positive cases for Covid-19 showed that the average age was 49 years, with a male prevalence. Important signs and symptoms were considered: fever (98\%), dry cough (76\%), dyspnea (55\%), myalgia or fatigue (44\%) and lymphopenia (63\%) " [10]. However, a larger study carried out in Wuhan, China on 
1099 confirmed positive patients stated that the mean age was 47 years and $41.9 \%$ were women, finding as symptoms: fever during admission (43.8\%); fever during hospitalization $(88.7 \%)$ and $(67.8 \%)$ had a cough [12]. We can say that the symptoms vary markedly from mild to severe in relation to the age of the patient, their health status (comorbidity), their weight and other factors.

Regarding prevention measures, there is a consensus in the protocols indicated in Peru by recognized institutions such as the Dental College that indicate as the main ones the use of a mask and physical distancing between person and person, in addition to hand washing and / or disinfecting them with alcohol. However, the stomatologist must take additional measures to minimize the risk of exposure during consultations and also during dental procedures typical of his profession (extractions, dressings, surgeries, etc.).

\section{Conclusion}

The presence of COVID-19 has had a great impact on the way in which routine and emergency dentistry practice is developed from now on, with important changes in the conditions of care for consultation and procedures and even leading to redefining what is considered an "urgency". Due to the fact that the potential risk of contagion for the stomatologist is very high thanks to the fact that said professional maintains direct and close contact with the oral mucosa and is enhanced during dental procedures where saliva and/or blood droplets are generated, This increases their exposure to the viral load of a positive patient, even if the patient is asymptomatic, it is a constant challenge to stay free of contagion, taking extreme measures of biosafety and self-care. The stomatologist must take a responsible and conscious position regarding the repercussions that COVID-19 has generated by taking the appropriate protection measures, both for him and for the patient, so it is suggested to take into account what is indicated by the Dental College of Peru and stay on the lookout for new knowledge regarding the new coronavirus.

\section{References}

1. López J, Cárdenas P, Giraldo G, Herrera Á (2020) Coronavirus-COVID 19: Beyond lung disease, what it is and what we know about the link with the cardiovascular system. Colombian Journal of Cardiology 27(3).

2. Ávila J (2020) Coronavirus CoVid-19; pathogenesis, prevention and treatment. Report. Leioa: College of Physiotherapists of the Community of Madrid, Department of Health.

3. Cortés M (2020) Coronavirus as a threat to public health. Rev Med Chil 148(1): 124-126.
4. Zhao WM, Song SH, Chen ML, Zou D, Ma LN, et al. (2020) The 2019 novel coronavirus resource. Yi Chuan 42(2): 212-221.

5. Dental College of Peru. Biosafety protocol for the dental surgeon during and after the COVID-19 pandemic.

6. Rubio Pérez I, Badia JM, Mora Rillo M, Martín Quirós A, García Rodríguez J, et al. (2020) COVID-19: Key Concepts for the Surgeon. Cir Esp 98(6): 310-319.

7. Pharmaceutical Colleges, Coronavirus: COVID-19. Technical report. Valencia, Department of Health.

8. Meng L, Hua F, Bian Z (2020) Coronavirus Disease 2019 (COVID-19): Emerging and Future Challenges for Dental and Oral Medicine. J Dent Res 99(5): 481-487.

9. van Doremalen N, Bushmaker T, Morris DH, Holbrook MG, Gamble A, et al. (2020) Aerosol and Surface Stability of SARS-CoV-2 as Compared with SARS-CoV-1. N Engl J Med 382(16): 1564-1567.

10. Belasco A, Fonseca C (2020) Coronavirus 2020. Rev Bras Enferm 73(2).

11. Lam TTY, Jia N, Zhang YW, Shum MHH, Jiang JF, et al. (2020) Identifying SARS-CoV-2-related coronaviruses in Malayan pangolins. Nature 583(7815): 282-285.

12. Guan W, Ni Z, Hu Y, Liang W, Ou C, et al. (2020) Clinical Characteristics of Coronavirus Disease 2019 in China. N Engl J Med 382(18): 1708-1720.

13. Lacoviello V (2020) COVID-19 (Novel Coronavirus). DynaMed 73(2).

14. Monitor AN (2020) Perú: Peru's COVID-19 Caseload Nears 100,000, Deaths Climb to 2,900. Thai News Service Group 73(2).

15. Valenzuela M (2020) Coronavirus and the dental office. J Oral Res 2(1): 14-19.

16. Collegiate Organization of Dentists of Spain. The new Coronavirus 2019-nCOV and the management of the dental patient.

17. Briggs $H$ (2020) Coronavirus: how the fence is tightened on the pangolin as a probable transmitter of the pathogen that causes COVID-19. BBC News World.

18. Dentistry in the times of the coronavirus-COVID-19. Int J Interdiscip 13(1): 2-2. 